\title{
Evidence for four- and three-wave interactions in solar type III radio emissions
}

\author{
G. Thejappa ${ }^{1}$, R. J. MacDowall ${ }^{2}$, and M. Bergamo ${ }^{1}$ \\ ${ }^{1}$ Department of Astronomy, University of Maryland, College Park, MD, USA \\ ${ }^{2}$ NASA/Goddard Space Flight Center, Greenbelt, USA
}

Correspondence to: G. Thejappa (thejappa.golla@nasa.gov)

Received: 10 April 2013 - Revised: 11 June 2013 - Accepted: 5 July 2013 - Published: 9 August 2013

\begin{abstract}
The high time resolution observations obtained by the STEREO/WAVES experiment show that in the source regions of solar type III radio bursts, Langmuir waves often occur as intense localized wave packets with short durations of only few ms. One of these wave packets shows that it is a three-dimensional field structure with $\frac{W_{\mathrm{L}}}{n_{\mathrm{e}} T_{\mathrm{e}}} \sim 10^{-3}$, where $W_{\mathrm{L}}$ is the peak energy density, and $n_{\mathrm{e}}$ and $T_{\mathrm{e}}$ are the electron density and temperature, respectively. For this wave packet, the conditions of the oscillating two-stream instability (OTSI) and supersonic collapse are satisfied within the error range of determination of main parameters. The density cavity, observed during this wave packet indicates that its depth, width and temporal coincidence are consistent with those of a caviton, generated by the ponderomotive force of the collapsing wave packet. The spectrum of each of the parallel and perpendicular components of the wave packet contains a primary peak at $f_{\text {pe }}$, two secondary peaks at $f_{\text {pe }} \pm f_{S}$ and a low-frequency enhancement below $f_{\mathrm{S}}$, which, as indicated by the frequency and wave number resonance conditions, and the fast Fourier transform (FFT)-based tricoherence spectral peak at $\left(f_{\mathrm{pe}}, f_{\mathrm{pe}}, f_{\mathrm{pe}}+f_{\mathrm{S}}, f_{\mathrm{pe}}-f_{\mathrm{S}}\right)$, are coupled to each other by the OTSI type of four-wave interaction ( $f_{\mathrm{pe}}$ is the local electron plasma frequency and $f_{\mathrm{S}}$ is the frequency of ion sound waves). In addition to the primary peak at $f_{\text {pe }}$, each of these spectra also contains a peak at $2 f_{\text {pe }}$, which as indicated by the frequency and wave number resonance conditions, and the wavelet-based bicoherence spectral peak at $\left(f_{\mathrm{pe}}, f_{\mathrm{pe}}\right)$, appears to correspond to the second harmonic electromagnetic waves generated as a result of coalescence of oppositely propagating sidebands excited by the OTSI. Thus, these observations for the first time provide combined evidence that (1) the OTSI and related strong turbulence processes play a significant role in the stabilization
\end{abstract}

of the electron beam, (2) the coalescence of the oppositely propagating up- and down-shifted daughter Langmuir waves excited by the OTSI probably is the emission mechanism of the second harmonic radiation, and (3) the Langmuir collapse follows the route of OTSI in some of the type III radio bursts.

Keywords. Solar physics, astrophysics, and astronomy (Radio emissions)

\section{Introduction}

The purpose of this paper is to present combined evidence for (1) the oscillating two-stream instability (OTSI) of Langmuir waves, which is the four-wave interaction $L_{1}+L_{2} \rightarrow$ $L_{\mathrm{U}}+L_{\mathrm{D}}$, where $L_{1}$ and $L_{2}$ are the beam-excited Langmuir waves, and $L_{\mathrm{U}}$ and $L_{\mathrm{D}}$ are the up- and down-shifted daughter Langmuir waves, respectively, and (2) the three-wave interaction $L_{\mathrm{U}}+L_{\mathrm{D}} \rightarrow T_{2 f_{\mathrm{pe}}}$, where $T_{2 f_{\mathrm{pe}}}$ is the electromagnetic wave at $2 f_{\mathrm{pe}}$. The high time resolution observations of the solar-type-III-burst-associated Langmuir waves are from the time domain sampler (TDS) of the STEREO/WAVES experiment (Bougeret et al., 2008). The unusual nature of the event analyzed in this study is that it occurs as a threedimensional structure, with the normalized peak energy density $\frac{W_{\mathrm{L}}}{n_{\mathrm{e}} T_{\mathrm{e}}}=\frac{\epsilon_{0} E_{\mathrm{L}}^{2}}{2 n_{\mathrm{e}} T_{\mathrm{e}}} \sim 10^{-3}$ satisfying the threshold conditions of OTSI and spatial collapse within the range of determination of main parameters $\left(n_{\mathrm{e}}\right.$ and $T_{\mathrm{e}}$ are the electron density and temperature, respectively, and $\epsilon_{0}$ is the permittivity of the free space). The peak amplitude of the wave packet $E_{\mathrm{L}}$ is determined using the peak amplitudes of the $E_{X}, E_{Y}$ and $E_{Z}$ waveforms as $E_{\mathrm{L}}=\sqrt{E_{X}^{2}+E_{Y}^{2}+E_{Z}^{2}}$. The analysis 
of the density fluctuations measured during the wave packet provides evidence for a density cavity, whose width, depth and time of occurrence are consistent with the caviton, generated by the ponderomotive force of the collapsing wave packet. The type III radio bursts, which are the radio signatures of solar-flare-accelerated electron beams in the corona and interplanetary medium (Kundu, 1965), are generally believed to be excited by the plasma mechanism (Ginzburg and Zheleznyakov, 1958), which involves the excitation of high levels of Langmuir waves by the flare-accelerated electron beams, and subsequent conversion of these Langmuir waves into electromagnetic waves at $f_{\mathrm{pe}}$ and $2 f_{\mathrm{pe}}$.

One of the outstanding issues is the identification of the nonlinear process behind the persistence of the electron beams over distances of $1 \mathrm{AU}$ and more against the quasilinear relaxation (Sturrock, 1964). It is suggested (Kaplan and Tsytovich, 1968) that the induced scattering by thermal ions when $T_{\mathrm{e}}=T_{\mathrm{i}}$, which acts as the electrostatic decay (ESD) of the Langmuir waves $(L)$ into daughter Langmuir $\left(L^{\prime}\right)$ and ion sound $(S)$ waves when $T_{\mathrm{e}}>T_{\mathrm{i}}$, can stabilize the beam by scattering the Langmuir waves from regions of resonance toward lower wave numbers $\left(T_{\mathrm{e}}\right.$ and $T_{\mathrm{i}}$ are the electron and ion temperatures, respectively). However, since the intensities of the beam-excited Langmuir waves in type III bursts are expected to be well above the threshold for the strong turbulence processes, the weak turbulence processes are argued to be not as efficient as OTSI (Papadopoulos et al., 1974; Goldstein et al., 1979; Smith et al., 1979) and related spatial collapse (Nicholson et al., 1978; Goldman et al., 1980), which can stabilize the beam by pumping the Langmuir waves from the resonance regions toward higher wave numbers.

A second unresolved issue is the identification of the emission mechanism of the second harmonic radiation, $T_{2 f_{\mathrm{pe}}}$. Ginzburg and Zheleznyakov (1958) proposed the three-wave interaction $L+L^{\prime} \rightarrow T_{2 f_{\mathrm{pe}}}$ as the probable emission mechanism, where $L$ is the beam-excited Langmuir wave, and $L^{\prime}$ is the oppositely propagating thermal Langmuir wave. However, if $L^{\prime}$ corresponds to thermal Langmuir waves, this process appears to yield very weak emissions. Melrose (1986) has suggested that if the nonlinear interactions $L \pm S \rightarrow L^{\prime}$ occur in type III bursts, then the daughter $L^{\prime}$ wave can serve as a good secondary wave, where $S$ is the ion sound wave. However, this process is shown to occur only when $T_{\mathrm{e}}>5 \times 10^{5} \mathrm{~K}$ for a narrowly peaked spectrum of Langmuir waves (Melrose, 1982). In the strong turbulence regime, Papadopoulos et al. (1974) proposed that the oppositely propagating sidebands excited by the OTSI are ideally suited to participate in wave-wave merging. The coherent emission by stable Langmuir solitons (Papadopoulos and Freund, 1978) and collapsing Langmuir solitons (Goldman et al., 1980) and the stochastic phase mixing of strongly turbulent Langmuir waves at long wavelengths (Kruchina et al., 1980) are a few other suggested emission mechanisms. Thus, there is no con- sensus about which of these mechanisms actually occurs in solar type III bursts. The preliminary evaluation performed using the ULYSSES observations appears to favor strong turbulence processes (Thejappa et al., 1996).

The in situ wave measurements have shown some signatures of the ESD in type III bursts (Lin et al., 1986; Gurnett et al., 1993; Hospodarsky and Gurnett, 1995; Thejappa and MacDowall, 1998; Thejappa et al., 2003; Henri et al., 2009). The high time resolution observations from the Fast Envelope Sampler (FES) of the ULYSSES/URAP experiment have provided some evidence for the strong Langmuir turbulence processes in type III burst sources (Kellogg et al., 1992; Thejappa et al., 1993, 1996, 1999; Thejappa and MacDowall, 1998, 2004). Thejappa et al. (2012a) had reported the high time resolution observations of a Langmuir wave packet obtained by the X-antenna of the STEREO spacecraft, and had shown that this wave packet satisfies the threshold conditions for OTSI, supersonic collapse as well as formation of collapsing envelope soliton, and its spectrum contains the signatures of OTSI, namely, the peaks corresponding to the beam-generated Langmuir waves, Stokes and anti-Stokes modes and ion sound waves, with frequencies and wave numbers satisfying the matching rules of the four-wave interaction. For the first time, Thejappa et al. (2012b) had applied the trispectral analysis technique on this wave packet and had shown that its spectral components are coupled to each other with a high degree of phase coherency (high tricoherence). Graham et al. (2012) analyzed this event using all the three components and argued that the OTSI may not be a viable process because the perpendicular component is stronger than the parallel component. However, these authors used only the fast Fourier transform (FFT) analysis, instead of using the higher-order spectral analysis techniques, and did not analyze the density fluctuations measured during this event.

The purpose of this paper is to present the results of (1) the proper analysis of three components of this wave packet, which uses the FFT, as well as the trispectral and bispectral analysis techniques, and (2) the analysis of the density fluctuations measured during the wave packet. We show that (1) the peak intensity of this wave packet satisfies the threshold condition of OTSI and supersonic collapse, as well as the formation of the collapsing wave packet within the error range of determination of main parameters, and the density cavity extracted from the low-frequency components provides evidence for the caviton generated by the ponderomotive force of the wave packet; (2) the spectra of the parallel as well as perpendicular components contain the sideband structures expected of OTSI, which satisfy the frequency and wave number resonance conditions, and, as shown by the trispectral analysis, are coupled to each other with a high degree of phase coherency; and (3) these spectra also contain, in addition to the primary peak at $f_{\mathrm{pe}}$, an intense peak at $2 f_{\text {pe }}$, which, as shown by the wavelet-based bispectral analysis, probably corresponds to the second harmonic 


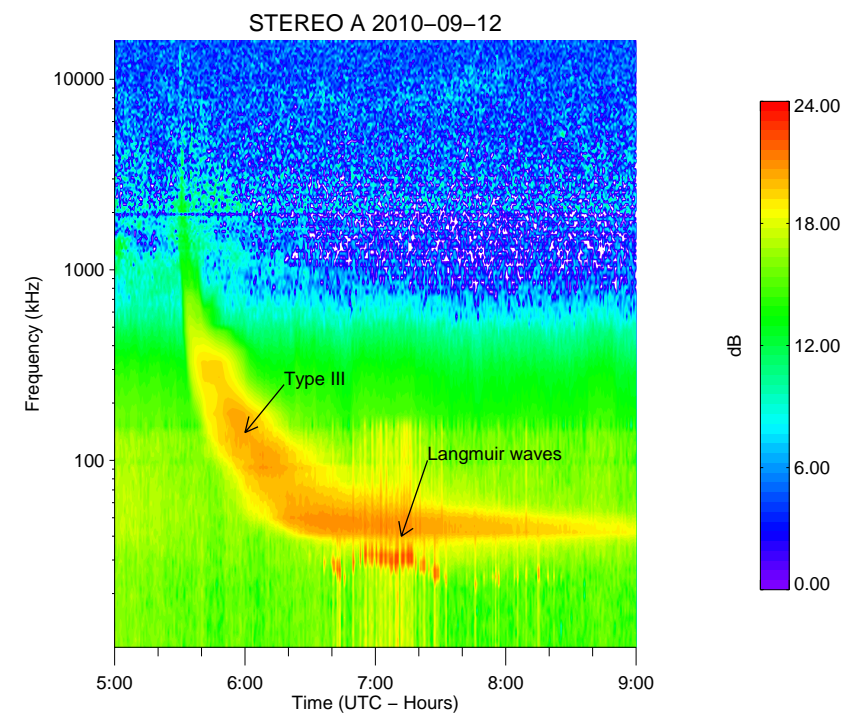

Fig. 1. Dynamic spectrum of a local type III radio burst (fast drifting emission from $\sim 5 \mathrm{MHz}$ down to $\sim 30 \mathrm{kHz}$ ) and associated Langmuir waves (non-drifting emissions in the frequency interval 27$32 \mathrm{kHz}$ ).

electromagnetic wave excited as a result of merging of oppositely propagating sidebands excited by the OTSI. These findings confirm that the observed wave packet provides unambiguous evidence for OTSI and spatial collapse as correctly concluded by Thejappa et al. (2012a,b). Furthermore, these findings also suggest that (1) the OTSI probably is responsible for the beam stabilization, (2) the coalescence of oppositely propagating up- and down-shifted Langmuir waves excited by OTSI probably is the excitation mechanism of the second harmonic emission, and (3) Langmuir collapse takes the route of OTSI in at least some type III bursts. In Sect. 2, we present the observations; in Sect. 3, we present the analysis; and in Sect. 4, we present the discussion and conclusions.

\section{Observations}

In Fig. 1, we present the dynamic spectrum of the type III burst and its associated Langmuir waves, obtained by the STEREO/WAVES experiment (Bougeret et al., 2008). This type III event is identified as the local event because of its drift from $\sim 5 \mathrm{MHz}$ all the way to $2 f_{\mathrm{pe}} \sim 60 \mathrm{kHz}$. The fast negative frequency drifts of type III bursts are usually attributed to the velocities of the electron beams, moving radially outward along the Parker's spiral magnetic field lines. Papagiannis (1970) has derived an expression relating these frequency drifts to the velocities $v_{\mathrm{b}}$ of the electron beams, assuming that the mode of emission is the second harmonic, and that the electron density $n_{\mathrm{e}}$ is described by the Radio Astronomy Explorer (RAE) model (Fainberg and Stone, 1971). Using this expression, we have fitted a curve for the fre-
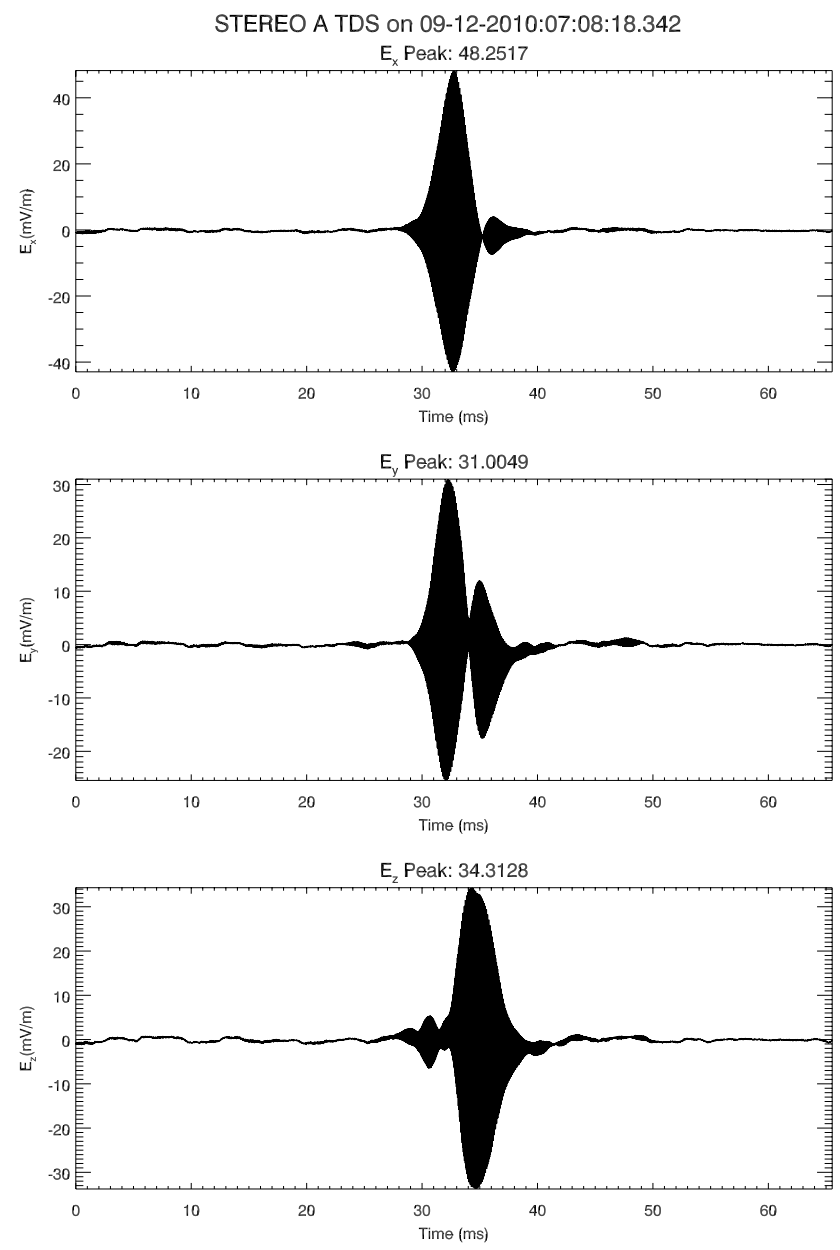

Fig. 2. The $E_{x}, E_{y}$ and $E_{z}$ components of the Langmuir wave packet detected by the $X, Y$ and $Z$ antennas. The waveforms are in the spacecraft frame of reference.

quency drift of the type III burst, and derived the beam speed $v_{\mathrm{b}} \sim 0.22 c$ ( $c$ is the velocity of light). Since the pitch angle scattering increases the path length traveled by the type III electrons by a factor of $\alpha=1.3$ to 1.7 (Lin et al., 1973; Alvarez et al., 1975), the beam speed $v_{\mathrm{b}}$ is corrected accordingly; i.e., $v_{\mathrm{b}}$ in the present case probably ranges from $\sim 0.29 c$ to $\sim 0.37 c$. This method has been used to estimate the beam speeds in several of our studies (Thejappa et al., 2012c, 2013a,b).

The non-drifting emissions in the $27-32 \mathrm{kHz}$ interval in Fig. 1 are the Langmuir waves. These Langmuir waves, which usually occur as intense isolated bursts, are resolved into intense waveforms by the TDS (Kellogg et al., 2009) of the SWAVES experiment. The TDS gathers the A/C electric field signals on three channels simultaneously connected to three orthogonal antennas and to a fourth pseudodipole channel obtained by taking the difference of any two monopoles. A typical waveform contains 16384 samples, acquired at the rate of 250000 samples per second (a time step 


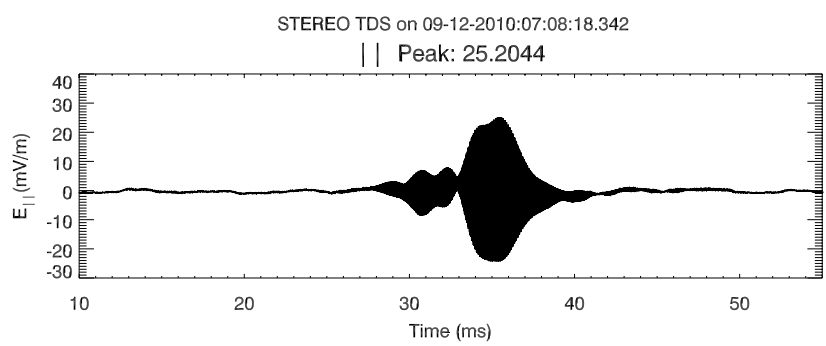

$\perp 1$ Peak: 50.0965

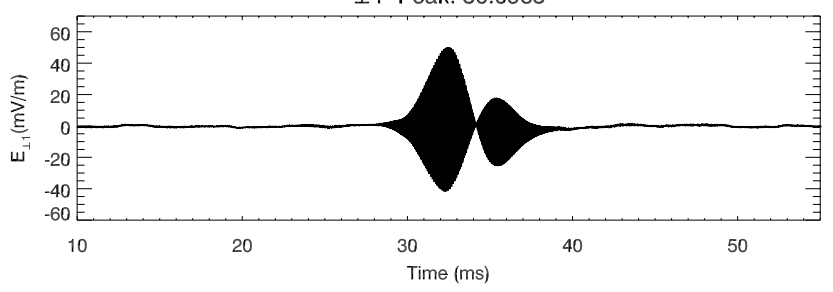

$\perp 2$ Peak: 31.2613

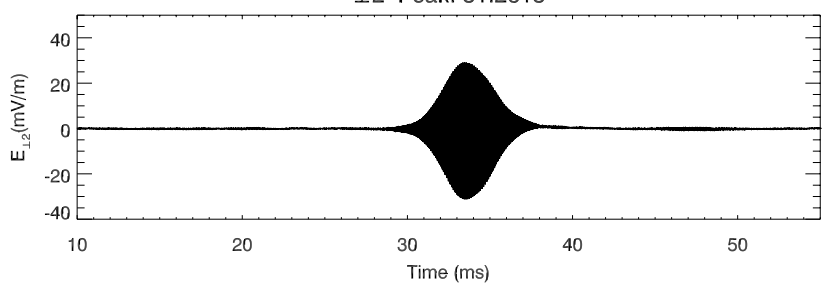

Fig. 3. The parallel (panel a) and perpendicular (panels b and c) electric field components of the Langmuir wave packet with respect to the magnetic field. The normalized vectors of magnetic field and solar wind velocity used in this transformation are $\boldsymbol{B}=[-0.60918,-0.39479,-0.69389]$ and $\boldsymbol{v}_{\mathbf{s w}}=$ $[0.99335,-0.10097,-0.055360]$, respectively. The angle between the magnetic field and solar wind velocity in this case is $\sim 122^{\circ}$.

of $4 \mu$ s for a total duration of $65 \mathrm{~ms}$ ). The transfer matrix given by Macher et al. (2007) is usually used to convert the potential into electric field measurements. This takes the effective antenna lengths into account. In Fig. 2, we present the $E_{X}, E_{Y}$ and $E_{Z}$ components of the most intense wave packet captured by the TDS during the type III event of Fig. 1. The peak amplitudes of these waveforms are 48.3, 31 and $\sim 34.3 \mathrm{mV} \mathrm{m}^{-1}$, respectively.

We have converted the $E_{X}, E_{Y}$ and $E_{Z}$ components from the spacecraft into the $\boldsymbol{B}$-aligned coordinate system with $\boldsymbol{B}$ along the $z$ axis, $\boldsymbol{B} \times \boldsymbol{v}_{\text {sw }}$ along the $x$ axis, and $\boldsymbol{B} \times\left(\boldsymbol{v}_{\mathbf{s w}} \times \boldsymbol{B}\right)$ along the $y$ axis $\left(\boldsymbol{v}_{\mathbf{s w}}\right.$ is the solar wind velocity). In the present case, the unit vectors of these quantities are $\boldsymbol{B}=(-0.60918,-0.39479,-0.69389)$ and $\boldsymbol{v}_{\mathbf{s w}}=$ $(0.99335,-0.10097,-0.055360)$, and the angle between them is $\sim 122^{\circ}$. In Fig. 3, we present these transformed waveforms, the parallel component in Fig. 3a, and the perpendicular components in Figs. $3 \mathrm{~b}$ and c. The peak electric field strengths of the parallel and perpendicular components are $\sim 25.2$, and $\sim 50.1$ and $\sim 31.3 \mathrm{mV} \mathrm{m}^{-1}$, respectively, which indicate that the observed Langmuir waveform is a 3-D wave packet.

In Fig. 4, we present the superposition of the spectra of the parallel and perpendicular components. The total spectrum of each of these components from 0 to $65 \mathrm{kHz}$ (Fig. 4a) clearly shows peaks at (approximate) 30 and $60 \mathrm{kHz}$, where the base frequency, $30 \mathrm{kHz}$, corresponds to Langmuir waves excited at the local electron plasma frequency, $f_{\mathrm{pe}}$, and the $60 \mathrm{kHz}$ corresponds to the second harmonic. As shown from these spectra, the second harmonic is weaker than the fundamental, which is typical of the natural signals (Walker et al., 2002; Kellogg et al., 2010; Malaspina et al., 2010). The superposition of the logarithmic spectra in the frequency interval from 29 to $31 \mathrm{kHz}$ (Fig. 4b) shows an intense peak $(L)$ of the beam-excited Langmuir waves at $f_{\mathrm{pe}} \sim 30 \mathrm{kHz}$, a Stokes peak $(D)$ at $\sim 29.5 \mathrm{kHz}$ and an anti-Stokes peak $(U)$ at $\sim 30.5 \mathrm{kHz}$. The linear spectra from 0 to $1.4 \mathrm{kHz}$ (Fig. $4 \mathrm{c}$ ) exhibit low-frequency enhancements below $\sim 400 \mathrm{~Hz}$, corresponding probably to ion sound waves.

For electron temperature $\left(T_{\mathrm{e}}\right)$, we assign a typical value of $10^{5} \mathrm{~K}$; the measurements of $T_{\mathrm{e}}$ are not available. Assuming that the intense peak $(L)$ in the spectra of parallel and perpendicular components corresponds to Langmuir waves excited at the local electron plasma frequency $f_{\mathrm{pe}} \sim 30 \mathrm{kHz}$ (Fig. 4), we estimate $n_{\mathrm{e}} \sim 1.1 \times 10^{7} \mathrm{~m}^{-3}$. These values yield Debye length, $\lambda_{\mathrm{De}}=69 T_{\mathrm{e}}^{1 / 2} n_{\mathrm{e}}^{-1 / 2} \sim 6.6 \mathrm{~m}$. As far as the wave numbers of Langmuir waves, $k_{\mathrm{L}}=\frac{2 \pi f_{\mathrm{pe}}}{v_{\mathrm{b}}}$, are concerned, they can be estimated to range from $\sim 2.2 \times 10^{-3} \mathrm{~m}^{-1}$ to $\sim 1.7 \times$ $10^{-3} \mathrm{~m}^{-1}$; i.e., $k_{\mathrm{L}} \lambda_{\text {De }}$ range from $1.1 \times 10^{-2}$ to $1.5 \times 10^{-2}$ for the beam speeds ranging from $\sim 0.29 c$ to $\sim 0.37 c$. The STEREO/PLASTIC experiment (Galvin et al., 2008) has measured $v_{\mathrm{sw}} \sim 450 \mathrm{~km} \mathrm{~s}^{-1}$.

\section{Analysis}

An isotropic plasma supports two eigenmodes, Langmuir and ion sound modes. The dispersion relation for the Langmuir waves can be written as

$\omega_{\mathrm{L}}=\omega_{\mathrm{pe}}\left(1+\frac{3}{2} k_{\mathrm{L}}^{2} \lambda_{\mathrm{De}}^{2}\right)$,

where $\omega_{\mathrm{pe}}=2 \pi f_{\mathrm{pe}}$. In the weak turbulence regime, each frequency $\omega_{\mathrm{L}}$ corresponds to one and only one wave number $k_{\mathrm{L}}$. In a statistical sense, the Langmuir turbulence in this regime is homogeneous, and the energy transfer can occur only toward $k_{\mathrm{L}} \rightarrow 0$. The dispersion relation of the ion sound waves is

$\omega_{S}=k_{S} c_{S}$

where $k_{S}$ and $c_{S}$ are the wave number and speed of the ion sound waves, respectively. When the energy density of Langmuir waves $W_{\mathrm{L}}$ is very low, the Langmuir and ion sound waves are uncoupled, and they become strongly coupled to 

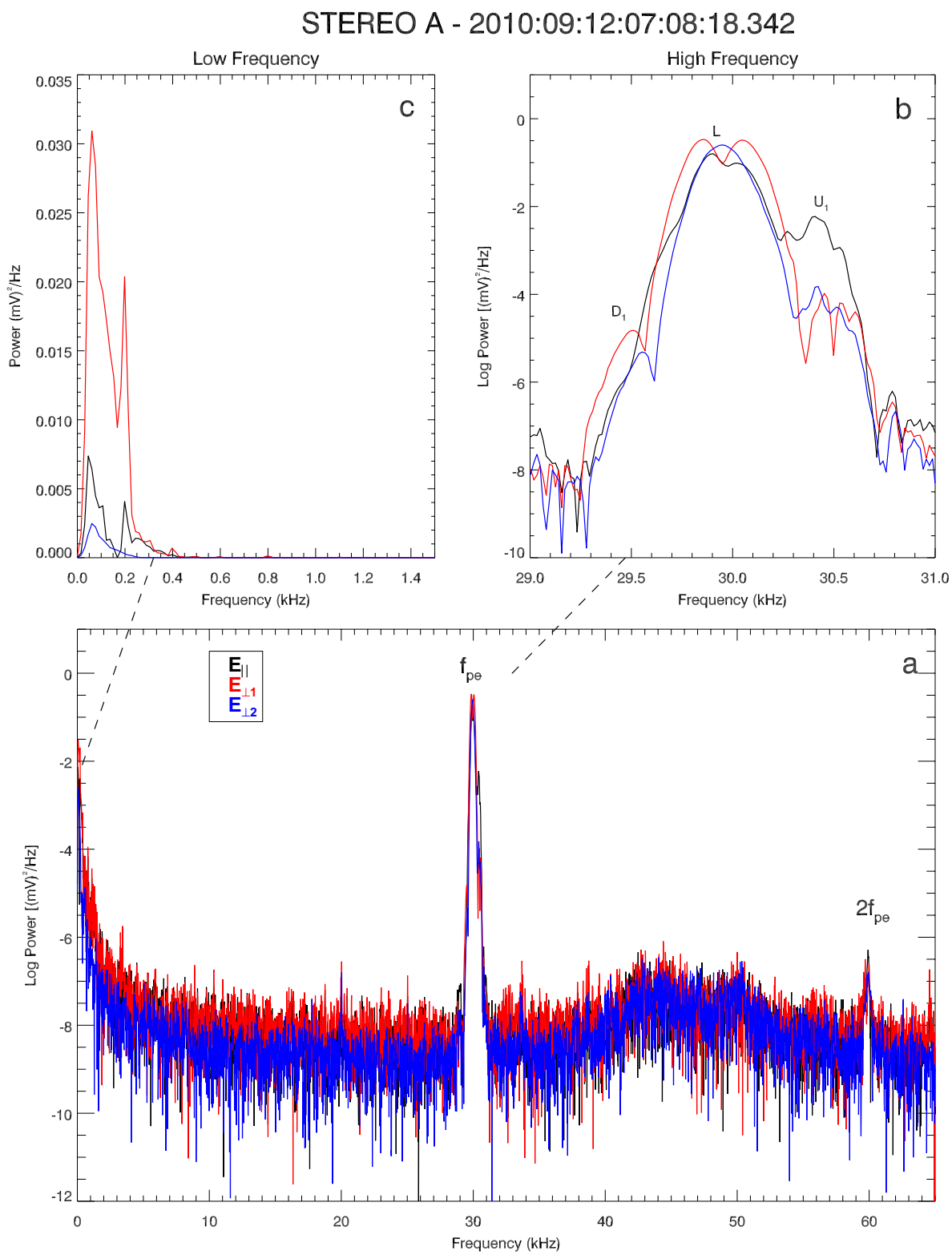

Fig. 4. (a) The superposition of the complete spectra of the parallel and perpendicular components of the wave packet; (b) the narrow spectrum around $f \sim f_{\mathrm{pe}} \sim 30 \mathrm{kHz}$, where $\mathrm{L}, \mathrm{D}$, and $\mathrm{U}$ correspond to the beam-excited Langmuir wave, down-shifted sideband at $\sim 29.5 \mathrm{kHz}$, and up-shifted sideband at $\sim 30.5 \mathrm{kHz}$, respectively; and (c) the low-frequency spectrum: the enhancement below $400 \mathrm{~Hz}$ corresponds to ion sound waves.

each other if $W_{\mathrm{L}}$ exceeds a certain threshold. The force behind this coupling is the ponderomotive force $\boldsymbol{F}_{\mathrm{p}}$ exerted by the Langmuir waves on the ambient plasma (Nezlin, 1993)

$\boldsymbol{F}_{\mathrm{p}}=-\nabla W_{\mathrm{L}}$,

which creates density cavities. From the equilibrium condition between the ponderomotive pressure and excess pressure of the plasma, one can estimate the relative depth of the density cavity as (Nezlin, 1993)

$\frac{\delta n_{\mathrm{e}}}{n_{\mathrm{e}}}=\frac{W_{\mathrm{L}}}{n_{\mathrm{e}} T_{\mathrm{e}}}$.
We can verify this relationship by extracting the density fluctuations from the low-frequency components of the wave packet. The density fluctuations $\frac{\delta n_{\mathrm{e}}}{n_{\mathrm{e}}}$ in the vicinity of the spacecraft and the change in the spacecraft potential $\delta \Phi_{\mathrm{sc}}$ are related as (Henri et al., 2011)

$\frac{\delta n_{\mathrm{e}}}{n_{\mathrm{e}}}=\frac{1}{T_{\mathrm{ph}}[e V]} \delta \Phi_{\mathrm{sc}}[V]$,

where $T_{\mathrm{ph}}=3 \mathrm{eV}$ is the photo electron temperature. Using this equation, we have converted the voltages observed on the $X, Y$ and $Z$ antennas in the frequency band from 0 to 


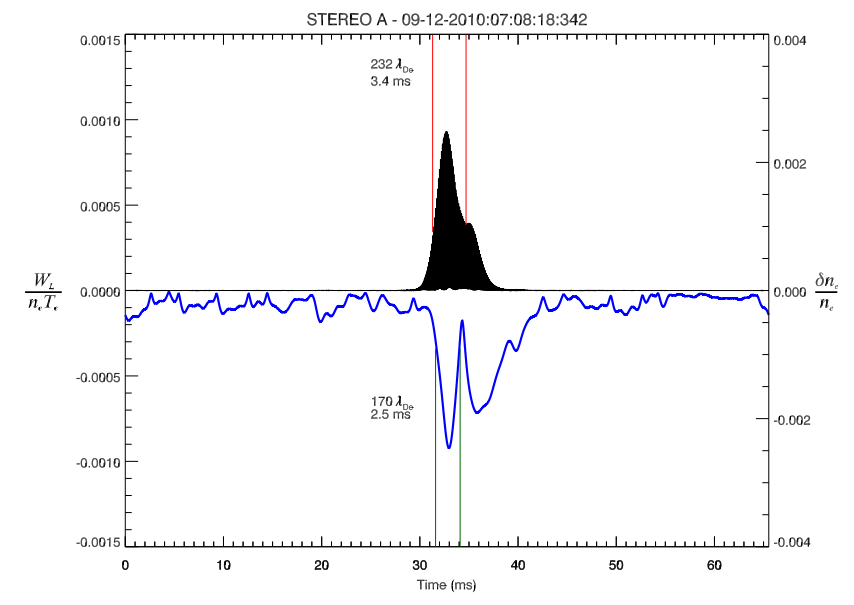

Fig. 5. The simultaneous observations of the collapsing Langmuir wave packet plotted in terms of normalized energy density, $\frac{W_{\mathrm{L}}(t)}{n_{\mathrm{e}} T_{\mathrm{e}}}$, and the density fluctuations, $\frac{\delta n_{\mathrm{e}}(t)}{n_{\mathrm{e}}}$.

$1 \mathrm{kHz}$ into density fluctuations, $\frac{\delta n_{\mathrm{e}}}{n_{\mathrm{e}}}$. We estimate the peak amplitude of the three-dimensional wave packet as $E_{\mathrm{L}}=$ $\sqrt{E_{x}^{2}+E_{y}^{2}+E_{z}^{2}}$, and calculate its normalized peak energy density, $\frac{W_{\mathrm{L}}}{n_{\mathrm{e}} T_{\mathrm{e}}}=\frac{\epsilon_{0} E_{\mathrm{L}}^{2}}{2 n_{\mathrm{e}} T_{\mathrm{e}}}$. We show the time variations of the measured $\frac{W_{\mathrm{L}}}{n_{\mathrm{e}} T_{\mathrm{e}}}$ and $\frac{\delta n_{\mathrm{e}}}{n_{\mathrm{e}}}$ in the top and bottom panels of Fig. 5 . As seen from these time profiles, $\frac{W_{\mathrm{L}}}{n_{\mathrm{e}} T_{\mathrm{e}}}$ and $\frac{\delta n_{\mathrm{e}}}{n_{\mathrm{e}}}$ show very good one-to-one correspondence. As far as the $\frac{1}{e}$-power spatial scales of the density cavity and $E_{\mathrm{L}}^{2}$-pocket are concerned, they are estimated as $\sim 170 \lambda_{\text {De }}$ and $\sim 232 \lambda_{\text {De }}$, respectively, for the $\frac{1}{e}$-power timescales of $\sim 2.5 \mathrm{~ms}$ and $\sim 3.4 \mathrm{~ms}$, respectively, and for the solar wind speed of $v_{\mathrm{sw}}=450 \mathrm{~km} \mathrm{~s}^{-1}$. Thus, the spatial scales and timescales also show reasonable agreement with each other. As far as the depth of the density cavity $\frac{\delta n_{\mathrm{e}}}{n_{\mathrm{e}}}$ is concerned, it is $\sim 10^{-3}$, being in good agreement with the peak value of $\frac{W_{\mathrm{L}}}{n_{\mathrm{e}} T_{\mathrm{e}}} \sim 10^{-3}$. This indicates that the observed density cavity probably is the caviton created by the ponderomotive force of the wave packet.

Thus, if

$$
\frac{W_{\mathrm{L}}}{n_{\mathrm{e}} T_{\mathrm{e}}}>\alpha\left(k_{\mathrm{L}} \lambda_{\mathrm{De}}\right)^{2},
$$

then the weak turbulence approximation is no longer valid, where $\alpha \simeq 1$ according to Zakharov (1972) and Zakharov (1985). Extending the heuristic arguments of Sagdeev (1979), Shapiro and Shevchenko (1985) assigned a value of 3 for $\alpha$. Thus, if Eq. (6) is satisfied, the OTSI and spatial collapse become important (Zakharov, 1972), and there won't be one-to-one correspondence between $\omega_{\mathrm{L}}$ and $k_{\mathrm{L}}$, since $\frac{W_{\mathrm{L}}}{n_{\mathrm{e}} T_{\mathrm{e}}}$ determines the bandwidth. Since the Langmuir oscillations get trapped in the self-generated density cavities, the turbulence becomes highly inhomogeneous, consisting of localized collapsing soliton-caviton pairs. The energy trans- fer in this case occurs in the direction of higher values of $k_{\mathrm{L}}$. In the present case, Eq. (6) is satisfied even for $\alpha \simeq 3$ within the error range of determination of main parameters, since $\frac{W_{\mathrm{L}}}{n_{\mathrm{e}} T_{\mathrm{e}}} \sim 10^{-3}$ and $\left(k_{\mathrm{L}} \lambda_{\mathrm{De}}\right)^{2}$ ranges from $\sim 1.3 \times 10^{-4}$ to $\sim 2.1 \times 10^{-4}$. The threshold for the supersonic collapse (Zakharov, 1972)

$\frac{W_{\mathrm{L}}}{n_{\mathrm{e}} T_{\mathrm{e}}} \geq \frac{m_{\mathrm{e}}}{m_{\mathrm{i}}}$

is also satisfied in the present case, since $\frac{W_{\mathrm{L}}}{n_{\mathrm{e}} T_{\mathrm{e}}} \sim 10^{-3}$ and $\frac{m_{\mathrm{e}}}{m_{\mathrm{i}}} \sim 5.5 \times 10^{-4}\left(m_{\mathrm{e}}\right.$ and $m_{\mathrm{i}}$ are the electron and ion masses, respectively). This condition signifies that the compression due to ponderomotive force and self-focusing overwhelms the wave packet spreading due to dispersion.

The criterion for the formation of the collapsing wave packet is (Thornhill and ter Haar, 1978; Gurnett et al., 1981)

$\frac{W_{\mathrm{L}}}{n_{\mathrm{e}} T_{\mathrm{e}}} \geq\left(\Delta k \lambda_{\mathrm{De}}\right)^{2}$,

where $\left(\Delta k=\frac{2 \pi}{S}\right)$ is the wave number characteristic of the envelope. This condition is also satisfied since the observed $\frac{W_{L}}{n_{\mathrm{e}} T_{\mathrm{e}}} \sim 10^{-3}$ is greater than $\left(\Delta k \lambda \lambda_{\mathrm{De}}\right)^{2} \sim 1.2 \times 10^{-4}$ estimated for the spatial scale $S$ of $\sim 232 \lambda \mathrm{De}$. In the present case, the observed field structure refers to a 3 -D soliton.

\subsection{Four-wave interactions}

The OTSI is a four-wave interaction process, in which two beam-excited Langmuir waves (pump waves) with frequencies and wave numbers $\left(f_{\mathrm{L}}, k_{\mathrm{L}}\right)$ non-linearly couple to upand down-shifted Langmuir sidebands with $\left(f_{\mathrm{U}}, k_{\mathrm{U}}\right)$ and $\left(f_{\mathrm{D}}\right.$, $\left.k_{\mathrm{D}}\right)$ through a purely growing ion sound mode with $\left(f_{S}, k_{S}\right)$. This parametric coupling process imposes the following frequency, wave number and phase matching conditions:

$$
\begin{aligned}
& 2 f_{\mathrm{L}}=f_{\mathrm{D}}+f_{\mathrm{U}} \\
& 2 k_{\mathrm{L}}=k_{\mathrm{D}}+k_{\mathrm{U}} \\
& 2 \phi_{\mathrm{L}}=\phi_{\mathrm{D}}+\phi_{\mathrm{U}},
\end{aligned}
$$

where the subscripts L, D and U correspond to the beamexcited Langmuir, down- and up-shifted sidebands, respectively.

In the present case, the spectra of the parallel and perpendicular components (Fig. 4) show spectral signatures of strong Langmuir wave peaks with upper and lower sidebands, together with low-frequency enhancements, which can be interpreted in terms of the pump wave, and the nonlinearly excited daughter sidebands and low-frequency waves, respectively. For both parallel and perpendicular components, the frequency matching condition $2 f_{\mathrm{L}}=f_{\mathrm{D}}+f_{\mathrm{U}}$ is easily satisfied, since the frequency shifts $\Delta f$ of the downand up-shifted sidebands are symmetric with respect to the Langmuir wave pump, being $\sim 500$ and $\sim 500 \mathrm{~Hz}$, respectively. These frequency differences $\Delta f=\left|f_{\mathrm{L}}-f_{\mathrm{U}, \mathrm{D}}\right|$ are also 
in good agreement with the frequencies of the ion sound waves of $<400 \mathrm{~Hz}$. Using the expression for the Doppler shift, $k_{S}=\frac{2 \pi f_{S}}{v_{\mathrm{sw}}}$, the wave numbers of the ion sound waves $k_{S} \lambda_{\text {De }}$ can be estimated as $\simeq 0.04$ for $f_{S}=400 \mathrm{~Hz}$ and $v_{\mathrm{sw}}=$ $450 \mathrm{~km} \mathrm{~s}^{-1}$. As far as the matching condition $k_{\mathrm{U}, \mathrm{D}}=k_{\mathrm{L}} \pm k_{S}$ is concerned, it is reasonably satisfied, yielding $\left|k_{\mathrm{U}, \mathrm{D}}\right| \simeq\left|k_{S}\right|$, since $k_{\mathrm{L}} \lambda_{\mathrm{De}}$, which ranges from $1.1 \times 10^{-2}$ to $1.5 \times 10^{-2}$, is three to four times less than $k_{S} \lambda_{\mathrm{De}} \sim 0.04$.

\section{Trispectral analysis}

The trispectrum, which is the fourth-order spectrum of the wave packet, decomposes the wave packet's kurtosis over frequencies. The trispectral analysis is an extremely useful tool to extract the signatures of the OTSI type of four-wave interactions from the waveform data (Kravtchenko-Berejnoi et al., 1995). The expression for the cumulant-based trispectrum is given by (Kravtchenko-Berejnoi et al., 1995)

$T(1,2,3)=E\left[X_{1} X_{2} X_{3}^{*} X_{4}^{*}\right]-N(1,2,3,4)$,

where $X_{1}, X_{2}, X_{3}$ and $X_{4}$ are the complex Fourier components of the wave packet, and $f_{1}, f_{2}, f_{3}$ and $f_{4}$ are the corresponding frequencies, respectively, and $N(1,2,3,4)=E\left[X_{1} X_{2}\right] E\left[X_{3}^{*} X_{4}^{*}\right]+E\left[X_{1} X_{3}^{*}\right] E\left[X_{2} X_{4}^{*}\right]+$ $E\left[X_{1} X_{4}^{*}\right] E\left[X_{2} X_{3}^{*}\right], f_{4}=f_{1}+f_{2}-f_{3}$, and $E[]$ is the expectation operator. The expression for the tricoherence, which is the normalized trispectrum, can be written as follows (Kravtchenko-Berejnoi et al., 1995):

$t^{2}(1,2,3)=\frac{|T(1,2,3)|^{2}}{\left(E\left[\left|X_{1} X_{2} X_{3}^{*} X_{4}^{*}\right|\right]+|N(1,2,3,4)|\right)^{2}}$.

This quantifies the fraction of the total product of powers at the frequency quartet, $\left(f_{1}, f_{2}, f_{3}, f_{1}+f_{2}-f_{3}\right)$, which is due to the cubicly phase-coupled modes. The method of periodograms is usually used to estimate the tricoherence (see Kravtchenko-Berejnoi et al., 1995, for details). The tricoherence estimator is symmetric with respect to permutations of its arguments $f_{1}, f_{2}$ and $f_{3}$. The principal domain for the interaction of the type $f_{1}+f_{2}=f_{3}+f_{4}$ is determined as (Kravtchenko-Berejnoi et al., 1995) $0 \leq f_{1} \leq f_{\mathrm{N}}$, $0 \leq f_{2} \leq f_{1}, 0 \leq f_{3} \leq f_{2}$, and $f_{3} \leq f_{1}+f_{2}-f_{3} \leq f_{\mathrm{N}}$, where $f_{\mathrm{N}}$ is the Nyquist frequency.

In this study, we have calculated the tricoherence spectrum as a function of three frequencies using $N=1000(0.004 \mathrm{~s})$ and $M=16$ with a Hamming window, where $N$ is the segment length, and $M$ is the number of segments. We display the results obtained for the waveforms of the parallel as well as perpendicular components in Fig. 6. Each of these spectra is the cross-section at $f_{\mathrm{L}}=30 \mathrm{kHz}$ of the three-dimensional tricoherence spectrum. The tricoherence spectral peaks seen at $(\sim 30.5, \sim 30, \sim 30) \mathrm{kHz}$ in these spectra correspond to four-wave interaction $(30+30 \rightarrow 30.5+29.5) \mathrm{kHz}$. The peak tricoherences in the case of $E_{\|}$and $E_{\perp 1}$ waveforms, as seen from the top two panels, are $\sim 0.6$ and $\sim 0.45$, respectively.
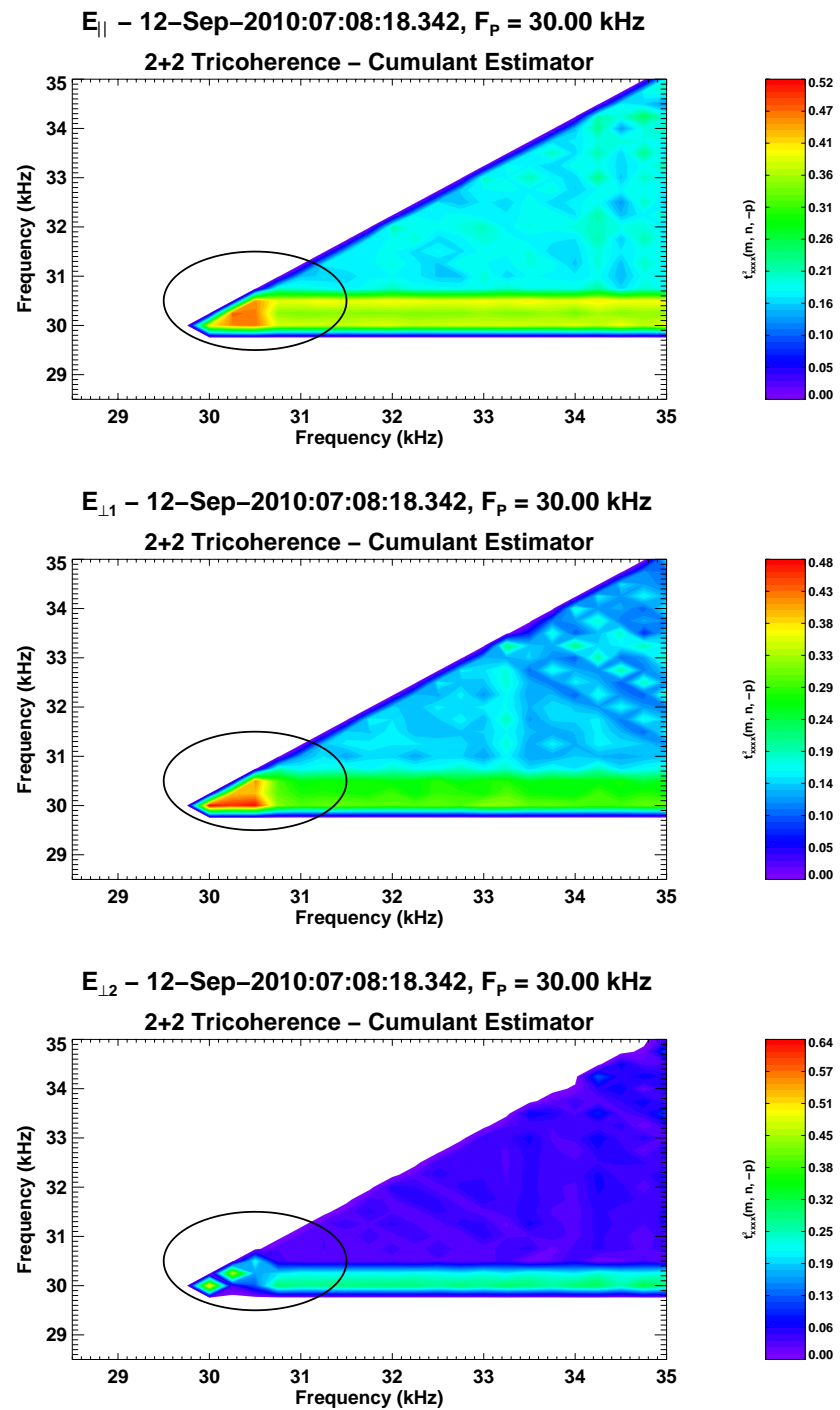

Fig. 6. The tricoherence spectra $t^{2}\left(F_{k}, F_{l}, F_{\mathrm{p}}\right)$ of the parallel and perpendicular components of the wave packet. The tricoherences $t^{2}$ exhibit peak values of $\sim 0.6, \sim 0.45$ and $\sim 0.4$ at $(\sim 30.5, \sim 30$, $\sim 30) \mathrm{kHz}$, which quantifies the phase relation $2 \phi_{\mathrm{L}}=\phi_{\mathrm{D}}+\phi_{\mathrm{U}}$, where $\phi_{\mathrm{L}}, \phi_{\mathrm{D}}$ and $\phi_{\mathrm{U}}$ are the phases of the beam-excited Langmuir wave at $\sim 30 \mathrm{kHz}$, Stokes $(\sim 29.5 \mathrm{kHz})$ and anti-Stokes $(\sim$ $30.5 \mathrm{kHz}$ ) modes, respectively

One of the tricoherence spectral peaks of the $E_{\perp 2}$ waveform shown in the bottom panel with $t^{2} \sim 0.4$ also corresponds to the four-wave interaction $(30+30 \rightarrow 30.5+29.5) \mathrm{kHz}$. Thus, the computed tricoherence spectra of the parallel and perpendicular components of the waveform provide clear evidence for the phase coupling between the beam-excited and the upand down-shifted Langmuir waves. This indicates that the resonance condition $2 \phi_{\mathrm{L}}=\phi_{\mathrm{U}}+\phi_{\mathrm{D}}$ is satisfied in this case.

Thus, the observed spectral components satisfy all the resonance Eqs. (9) imposed by the OTSI. 


\subsection{Three-wave interactions}

Figure 4a shows that the total spectrum of each of the waveforms of the parallel and perpendicular components exhibits a primary peak at $f_{\text {pe }}$ corresponding to Langmuir waves, and a harmonic peak $2 f_{\text {pe }}$. If we interpret the harmonic peak in terms of the second harmonic Langmuir waves, the wavewave interactions between the Langmuir and second harmonic Langmuir wave are not allowed. On the other hand, if we interpret it in terms of the second harmonic electromagnetic wave emitted spontaneously either by the stable (Papadopoulos and Freund, 1978) or collapsing (Goldman et al., 1980) solitons, phase coherency between such a wave and the Langmuir wave is expected to be negligible, since the phases would be random for spontaneously excited emissions. Finally, if we interpret the spectral peak at $2 f_{\text {pe }}$ in terms of the second harmonic electromagnetic waves, excited by wavewave interactions, involving the coalescence of two oppositely propagating Langmuir waves as discussed by several authors (see, for example, Zheleznyakov, 1976)

$L_{1}+L_{2} \rightarrow T_{2 f_{\mathrm{pe}}}$

one expects $L_{1}, L_{2}$ and $T_{2} f_{\mathrm{pe}}$ to satisfy the frequency, wave number and phase resonance conditions imposed by this three-wave interaction. These resonance conditions are

$$
\begin{aligned}
& f_{1}+f_{2}=f_{t} \\
& k_{1}+k_{2}=k_{t} \\
& \phi_{1}+\phi_{2}=\phi_{t},
\end{aligned}
$$

where $f_{1}, f_{2}$ and $f_{t}, k_{1}, k_{2}$ and $k_{t}$, and $\phi_{1}, \phi_{2}$ and $\phi_{t}$ are the frequencies, wave numbers, and phases of the $L_{1}, L_{2}$ and $T_{2 f_{\mathrm{pe}}}$, respectively. If $L_{1}$ and $L_{2}$ correspond to $L_{\mathrm{U}}$ and $L_{\mathrm{D}}$ excited by the OTSI, then the frequency and wave number matching rules are easily satisfied, since $f_{\mathrm{U}}=f_{\mathrm{pe}}+f_{S}$ and $f_{\mathrm{D}}=f_{\mathrm{pe}}-f_{S}$, and therefore $f_{\mathrm{U}}+f_{\mathrm{D}}=2 f_{\mathrm{pe}}$. As far as the wave number resonance condition is concerned, since $\boldsymbol{k}_{\mathbf{U}}=$ $-\boldsymbol{k}_{\mathrm{D}}$ and $k_{t} \lambda_{\mathrm{De}} \sim \sqrt{3} \frac{2 \pi f_{\mathrm{pe}}}{c} \lambda_{\mathrm{De}} \sim 2.3 \times 10^{-3}$, the condition $k_{\mathrm{U}}+k_{\mathrm{D}}=k_{t}$ is also easily satisfied. As far as the phase coherence is concerned, its information can be extracted from the bispectral analysis of the wave packet.

\section{Bispectral analysis}

The wavelet-based bispectrum can be defined as (Henri et al., 2009)

$B\left(a_{1}, a_{2}\right)=\int W\left(a_{1}, \tau\right) W\left(a_{2}, \tau\right) W^{*}\left(a_{3}, \tau\right) d \tau$,

where $W(a, \tau)$ is the continuous wavelet transform (CWT) at scale $a$ and time $\tau$. This quantifies the phase coupling between the wavelet components of the waveform $x(t)$ with $a_{1}$, $a_{2}$ and $a_{3}$. The sum rule $a_{3}^{-1}=a_{1}^{-1}+a_{2}^{-2}$ can be interpreted as the coupling between wavelets of frequencies, such that
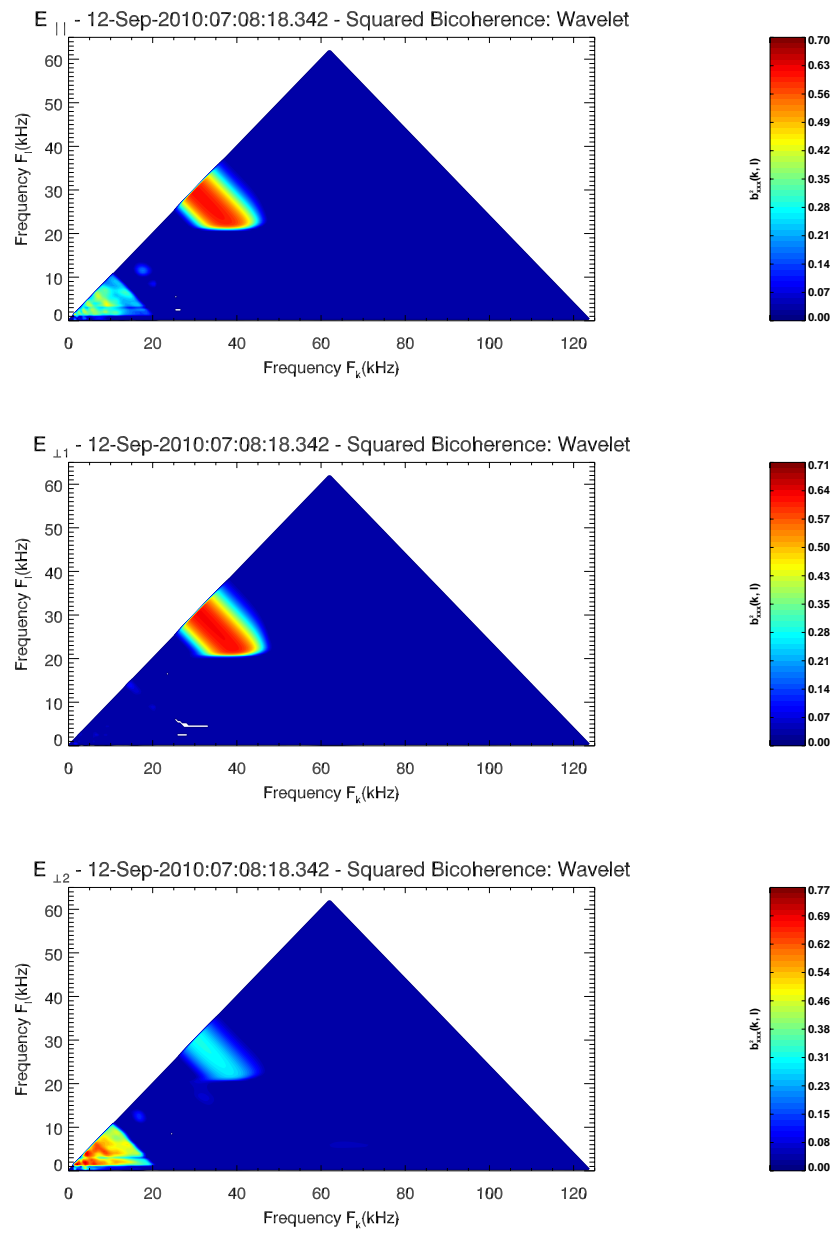

Fig. 7. The wavelet-based bicoherence spectra of the parallel and perpendicular components of the TDS event of Fig. 3. The bicoherence spectra of these components show peak values of $\sim 0.7, \sim 0.7$ and $\sim 0.3$ at $(\sim 30 \sim 30) \mathrm{kHz}$

$f_{3}=f_{1}+f_{2}$, where $f=f_{0} / a$. The bicoherence can be expressed as

$b^{2}\left(a_{1}, a_{2}\right)=\frac{\left|B\left(a_{1}, a_{2}\right)\right|^{2}}{\left[\int\left|W\left(a_{1}, \tau\right) W\left(a_{2}, \tau\right)\right|^{2} d \tau\right]\left[\int\left|W\left(a_{3}, \tau\right)\right|^{2} d \tau\right]}$.

Here the integrals should be replaced by the summations over $N$ points. The bispectrum and bicoherence are usually computed in the following frequency intervals:

$0<f_{1}<\frac{f_{\mathrm{N}}}{N}, f_{1}<f_{2}<\frac{f_{\mathrm{N}}}{2}-f_{1}$,

where $f_{\mathrm{N}}$ is the Nyquist frequency.

Using Eq. (15), we have computed the bicoherence spectra of the parallel and perpendicular components. As seen in Fig. 7, each of these computed bicoherence spectra contains an intense peak at $(30,30) \mathrm{kHz}$. The peak bicoherences of the parallel and perpendicular components of $\simeq 0.7$, and $\simeq 0.7$, 
and $\simeq 0.3$, respectively, provide unambiguous evidence for the nonlinear interaction $(30+30 \rightarrow 60) \mathrm{kHz}$. This suggests that the second harmonic peaks in the spectra of the parallel and perpendicular components probably are the harmonic electromagnetic waves at $2 f_{\text {pe }}$ excited from the coalescence of two oppositely propagating Langmuir waves. Thus, the spectral peak at $2 f_{\text {pe }}$ probably corresponds to the second harmonic electromagnetic wave excited from the three-wave interaction, $L_{1}+L_{2} \rightarrow T_{2 f_{\mathrm{pe}}}$, and the oppositely propagating waves probably correspond to the sidebands excited by the OTSI, which can collide head-on with each other and emit electromagnetic waves at $2 f_{\mathrm{pe}}$ as suggested by Papadopoulos et al. (1974).

\subsubsection{Flux comparison}

The power per unit area $S_{2 f_{\mathrm{pe}}}$ detected by a receiver within a bandwidth $\Delta f$ is (Robinson and Cairns, 1993)

$S_{2 f_{\mathrm{pe}}}=\epsilon_{0} c E_{2 f_{\mathrm{pe}}}^{2}$,

where $E_{2 f_{\mathrm{pe}}}$ is the peak amplitude of the electromagnetic wave. The expression for the total power radiated by the observed source located at a distance $R$ in the frequency range $\Delta f$ can be written as

$P_{2 f_{\mathrm{pe}}}=S_{2 f_{\mathrm{pe}}} R^{2} \Delta \Omega$,

where $\Delta \Omega$ is the solid angle subtended by the source at the receiver. The volume of the source is

$V=R^{2} \Delta R \Delta \Omega$

where $\Delta R \simeq R_{\mathrm{S}} \frac{\Delta f}{f}$ is the linear depth of the source and $R_{\mathrm{S}} \simeq 1 \mathrm{AU}$ is the distance of the source from the sun. Thus, the mean volume emissivity is

$J_{2 f_{\mathrm{pe}}}=\frac{P_{2 f_{\mathrm{pe}}}}{V \Delta \Omega} \simeq \frac{\epsilon_{0} c E_{2 f_{\mathrm{pe}}}^{2}}{\Delta \Omega \Delta R}$.

We have applied a bandpass filter of $2 \mathrm{kHz}$ width centered around $2 f_{\mathrm{pe}}=60 \mathrm{kHz}$ and determined the peak amplitudes of the $X, Y$, and $Z$ component waveforms. From these peak fields, we estimate $E_{2 f_{\mathrm{pe}}}=0.06 \mathrm{mV} \mathrm{m}^{-1}$ corresponding probably to the peak electric field of the second harmonic emission. For this peak electric field, and for $\Delta f=$ $2 \mathrm{kHz}$ and $\Delta \Omega=2 \pi$, we estimate the observed emissivity as $J_{2 f_{\mathrm{pe}}}=2.9 \times 10^{-22} \mathrm{~W} \mathrm{~m}^{-3} \mathrm{Sr}^{-1}$.

Papadopoulos et al. (1974) have derived an expression for the emissivity of the second harmonic radiation due to merging of the up- and down-shifted Langmuir waves excited by OTSI. In order to compare the observed emissivity with the predicted emissivity of the second harmonic emission from the observed Langmuir waves, this expression has been rewritten as (Gurnett et al., 1980)

$J_{2 f_{\mathrm{pe}}}=5.83 \times 10^{-12} \frac{E_{\mathrm{L}}^{4}}{\sqrt{n_{\mathrm{e}}}}$.
Thus, for $E_{\mathrm{L}} \simeq 56 \mathrm{mVm}^{-1}$ and $n_{\mathrm{e}}=1.1 \times 10^{7} \mathrm{~m}^{-3}$, we estimate the predicted emissivity as $J_{2 f_{\mathrm{pe}}} \sim$ $1.7 \times 10^{-20} \mathrm{~W} \mathrm{~m}^{-3} \mathrm{Sr}^{-1}$. Thus, with all the observational uncertainties, the emissivity estimated using the peak electric field at $2 f_{\text {pe }}$ reasonably agrees with the predicted emissivity estimated using the peak amplitude of the waveform.

\section{Discussion and conclusions}

The STEREO/WAVES experiment has observed an intense localized wave packet in the source region of a solar type III radio burst. Thejappa et al. (2012a) had reported the observations of the $X$ component of this wave packet and had shown that it is characterized by (1) a spectrum consisting of (a) a resonant peak at the local electron plasma frequency, $f_{\text {pe }}$, (b) Stokes peak at a frequency slightly lower than $f_{\text {pe }}$, (c) anti-Stokes peak at a frequency slightly higher than $f_{\text {pe }}$, and (d) low-frequency enhancement corresponding to ion sound fluctuations, which satisfy the resonance conditions of oscillating two-stream instability (OTSI); and by (2) peak intensity, which is well above the thresholds for OTSI, supersonic collapse and formation of collapsing envelope soliton. Thejappa et al. (2012b) have for the first time applied the trispectral analysis technique on this wave packet and showed that its spectral components are coupled to each other with a high degree of phase coherency. Graham et al. (2012) applying only the FFT analysis on all the three components of the wave packet, argued that the OTSI may not be a viable process.

As shown in this study, a thorough analysis of the wave packet using the FFT as well as the trispectral and bispectral analysis techniques, combined with the analysis of the density fluctuations measured during the wave packet, clearly shows that (1) the peak intensity of the wave packet determined within the error range of main parameters is above the threshold for oscillating two-stream instability (OTSI) as well as supersonic collapse; (2) the density fluctuations extracted from the low-frequency components provide evidence for a density cavity, generated most probably by the ponderomotive force of the wave packet; (3) the parallel as well as perpendicular components of the wave packet exhibit the characteristic spectral signatures of OTSI, namely, resonant peaks at local electron plasma frequency, $f_{\mathrm{pe}}$, Stokes peaks at a frequency slightly lower than $f_{\mathrm{pe}}$, anti-Stokes peaks at a frequency slightly higher than $f_{\text {pe }}$, and lowfrequency enhancements below a few hundred $\mathrm{Hz}$; (4) these spectral components satisfy the frequency and wave number resonance conditions of the OTSI type of four-wave interaction; and (5) the tricoherence spectra of the parallel as well as the perpendicular components contain the clear signatures of four-wave interaction $L_{1}+L_{2} \rightarrow L_{\mathrm{U}}+L_{\mathrm{D}}$, where $L_{1}$ and $L_{2}$ are the beam-excited Langmuir waves, and $L_{\mathrm{U}}$ and $L_{\mathrm{D}}$ are the up- and down-shifted sidebands, respectively. 
Furthermore, this analysis has clearly demonstrated that (1) the spectra of the parallel as well as the perpendicular components also contain harmonic peaks at $2 f_{\text {pe }}$ in addition to intense Langmuir-wave-associated peaks at $f_{\mathrm{pe}}$; (2) the waves of these spectral peaks satisfy the resonance conditions of the wave-wave merging $L_{\mathrm{U}}+L_{\mathrm{D}} \rightarrow T_{2 f_{\mathrm{pe}}}$; (3) the bicoherence spectra of these parallel and perpendicular components contain intense peaks at $\left(f_{\mathrm{pe}}, f_{\mathrm{pe}}\right)$, corresponding to the three-wave interaction $L_{\mathrm{U}}+L_{\mathrm{D}} \rightarrow T_{2 f_{\mathrm{pe}}}$, where $T_{2 f_{\mathrm{pe}}}$ is the second harmonic electromagnetic wave; and (4) the predicted and measured emissivities of second harmonic emissions agree with each other very well. Thus, these findings for the first time provide observational evidence for the threewave interaction $L_{\mathrm{U}}+L_{\mathrm{D}} \rightarrow T_{2 f_{\mathrm{pe}}}$ as the emission mechanism of the second harmonic emission.

It is important to note that the TDS events reported in our other studies (Thejappa et al., 2012c, 2013a,b) have also shown clear evidence for the linear as well as nonlinear regimes of OTSI. The TDS event of the present study is unique in the sense that it is the three-dimensional wave packet, which shows evidence for four-wave interaction (OTSI) as well as three-wave interaction $\left(L_{\mathrm{U}}+L_{\mathrm{D}} \rightarrow T_{2 f_{\mathrm{pe}}}\right)$ simultaneously.

In spite of uncertainties, such as the assumption of a typical value of $10^{5} \mathrm{~K}$ for the electron temperature, $T_{\mathrm{e}}$, and indirect estimation of the velocity of the electron beam $v_{\mathrm{b}}$ from the frequency drift of the type III radio burst, the findings of this study strongly support the following conclusions: (1) the observed wave packet provides unambiguous evidence for OTSI and spatial collapse as correctly concluded in Thejappa et al. (2012a,b), (2) the OTSI probably is responsible for the beam stabilization, (3) the coalescence of oppositely propagating up- and down-shifted Langmuir waves excited by OTSI probably is the excitation mechanism of the second harmonic emission, and (4) Langmuir collapse takes the route of OTSI in type III bursts.

Acknowledgements. The research of G. Thejappa is supported by the NASA Grants NNX09AB19G and NNX12AH47G. The SWAVES instruments include contributions from the Observatoire of Paris, University of Minnesota, University of California, Berkeley, and NASA/GSFC.

Guest Editor M. Balikhin thanks two anonymous referees for their help in evaluating this paper.

\section{References}

Alvarez, H., Lin, R. P., and Bame, S. J.: Fast solar electrons, interplanetary plasma and $\mathrm{km}$-wave type-III radio bursts observed from the IMP-6 spacecraft, Sol. Phys., 44, 485-501, 1975.

Bougeret, J.-L., Goetz, K., Kaiser, M. L., Bale, S. D., Kellogg, P. J., Maksimovic, M., Monge, N., Monson, S. J., Astier, P. L., Davy, S., Dekkali, M., Hinze, J. J., Manning, R. E., AguilarRodriguez, E., Bonnin, X., Briand, C., Cairns, I. H., Cattell, C. A., Cecconi, B., Eastwood, J., Ergun, R. E., Fainberg, J., Hoang,
S., Huttunen, K. E. J., Krucker, S., Lecacheux, A., MacDowall, R. J., Macher, W., Mangeney, A., Meetre, C. A., Moussas, X., Nguyen, Q. N., Oswald, T. H., Pulupa, M., Reiner, M. J., Robinson, P. A., Rucker, H., Salem, C., Santolik, O., Silvis, J. M., Ullrich, R., Zarka, P., and Zouganelis, I.: S/WAVES: The radio and plasma wave investigation on the STEREO Mission, Space Sci. Rev., 136, 487-529, 2008.

Fainberg, J. and Stone, R. G.: Type III solar radio burst storms observed at low frequencies, Sol. Phys., 17, 392-401, 1971.

Galvin, A. B., Kistler, L. M., Popecki, M. A., Farrugia, C. J., Simunac, K. D. C., Ellis, L., Möbius, E., Lee, M. A., Boehm, M., Carroll, J., Crawshaw, A., Conti, M., Demaine, P., Ellis, S., Gaidos, J. A., Googins, J., Granoff, M., Gustafson, A., Heirtzler, D., King, B., Knauss, U., Levasseur, J., Longworth, S., Singer, K., Turco, S., Vachon, P., Vosbury, M., Widholm, M., Blush, L. M., Karrer, R., Bochsler, P., Daoudi, H., Etter, A., Fischer, J., Jost, J., Opitz, A., Sigrist, M., Wurz, P., Klecker, B., Ertl, M., Seidenschwang, E., Wimmer-Schweingruber, R. F., Koeten, M., Thompson, B., and Steinfeld, D.: The Plasma and Suprathermal Ion Composition (PLASTIC) Investigation on the STEREO Observatories, Space Sci. Rev., 136, 437-486, 2008.

Ginzburg, V. L. and Zheleznyakov, V. V.: On the possible mechanisms of sporadic solar radio emission (radiation in an isotropic plasma), Sov. Astron., 2, 653-668, 1958.

Goldman, M. V., Reiter, G. F. and Nicholson, D. R.: Radiation from a strongly turbulent plasma: Application to electron beamexcited solar emissions, Phys. Fluids, 23, 388-401, 1980.

Goldstein, M. L., Smith, R. A., and Papadopoulos, K.: Nonlinear stability of solar type III radio bursts. Application to observations near 1 AU, Astrophys. J., 237, 683-695, 1979.

Graham, D. B., Cairns, I. H., Malaspina, D. M., and Ergun, R. E.: Evidence against the oscillating two-stream instability and spatial collapse of Langmuir waves in solar type III radio bursts, Astrophys. J. Lett., 753, L18-L21, 2012.

Gurnett, D. A., Anderson, R. R., and Tokar, R. L.: Plasma oscillations and the emissivity of type III radio bursts, in: Radio Physics of the Sun, edited by: Kundu, M. R. and Gergely, T. E., p. 369379, 1980.

Gurnett, D. A., Maggs, J. E., Gallagher, D. L., Kurth, W. S., Williams, D. J., and Scarf, F. L.: Parametric interaction and spatial collapse of beam driven Langmuir wave in the solar wind, J. Geophys. Res., 86, 8833-8841, 1981.

Gurnett, D. A., Hospodarsky, G. B., Kurth, W. S., Williams, D. J., and Bolton, S. J.: Fine structure of Langmuir waves produced by a solar electron event, J. Geophys. Res., 98, 5631-5637, 1993.

Henri, P., Briand, C., Mangeney, A., Bale, S. D., Califano, F., Goetz, K., and Kaiser, M.: Evidence for wave coupling in type III emissions, J. Geophys. Res., 114, A03103, doi:10.1029/2008JA013738, 2009.

Henri, P., Meyer-Vernet, N., Briand, C., and Donato, S.: Observations of Langmuir ponderomotive effects using the Solar TErrestrial RElations observatory spacecraft as a density probe, Phys. Plasmas, 18, 082308, doi:10.1063/1.3622667, 2011.

Hospodarsky, G. B. and Gurnett, D. A.: Beat-type Langmuir wave emissions associated with a type III solar radio burst: Evidence of parametric decay, Geophys. Res. Lett., 22, 1161-1164, 1995.

Kaplan, S. A. and Tsytovich, V. N.: Radio emission from beams of fast particles under cosmic conditions, Sov. Astron., 11, 956964, 1968. 
Kellogg, P. J., Goetz, K., Howard, R. L., and Monson, S.: Evidence for Langmuir wave collapse in the interplanetary plasma, Geophys. Res. Lett., 19, 1303-1306, 1992.

Kellogg, P. J., Goetz, K., Monson, S. J., Bale, S. D., Reiner, M. J., and Maksimovic, M.: Plasma wave measurements with STEREO S/WAVES: Calibration, potential model, and preliminary results, J. Geophys. Res., 114, A02107, doi:10.1029/2008JA013566, 2009

Kellogg, P. J., Goetz, K., and Monson, S. J.: Harmonics of Langmuir waves in the Earth's foreshock, J. Geophys. Res., 115, A06107, doi:10.1029/2009JA014635, 2010.

Kravtchenko-Berejnoi, V., Lefeuvre, F., Krasnoselskikh, V., and Lagoutte, D.: On the use of tricoherent analysis to detect nonlinear wave-wave interactions, Signal Processing, 42, 291-309, 1995.

Kruchina, E. N., Sagdeev, R. Z., and Shapiro, V. D.: Strong Langmuir turbulence as a source of radio emission, JETP Lett., 32, 419-423, 1980.

Kundu, M. R.: Solar Radio Astronomy, John Wiley and Sons Inc., 1965.

Lin, R. P., Evan, L. G., and Fainberg, J.: Simultaneous observations of fast solar electrons and type III radio burst emission near $1 \mathrm{AU}$, Astrophys. Lett., 14, 191-198, 1973.

Lin, R. P., Levedahl, W. K., Lotko, W., Gurnett, D. A., and Scarf, F. L.: Evidence for nonlinear wave-wave interactions in solar type III radio bursts, Astrophys. J., 308, 954-965, 1986.

Macher, W., Oswald, T. H., Fischer, G., and Rucker, H. O.: Rheometry of multiport spaceborne antennas including mutual antenna capacitances and application to STEREO/WAVES. Measurement Science and Technology, 18, 3731-3742, 2007.

Malaspina, D., Cairns, I. H., and Ergun, R. E.: The $2 f_{\mathrm{p}}$ radiation from localized Langmuir waves, J. Geophys. Res., 115, A01101, doi:10.1029/2009JA014609, 2010.

Melrose, D. B.: Fundamental emission for type III bursts in the interplanetary medium - The role of ion-sound turbulence, Solar Phys., 79, 173-185, 1982.

Melrose, D. B.: Instabilities in space and laboratory plasmas, Cambridge University Press, New York, 1986.

Nezlin, M. V.: Langmuir solitons in electron (plasma) beams, in: Physics of intense beams in plasmas, Philadelphia, Institute of Physics Publications, p. 186-249, 1993.

Nicholson, D. R., Goldman, M. V., Hoyang, P., and Weatherall. J. S.: Nonlinear Langmuir waves during type III solar radio bursts, Astrophys. J., 223, 605-619, 1978.

Papagiannis, M. D.: Studies of the Outer Corona through Space Radio Astronomy, Physics of the Solar Corona, Proceedings of the NATO Advanced Study Institute, held at Cavouri-Vouliagmeni, Athens, 6-17 September 1970, Dordrecht: Reidel, 1971, edited by: Macris, C. J., Astrophysics and Space Science Library, 27, p. 317,1970 .

Papadopoulos, K. and Freund, H. P.: Solitons and second harmonic radiation in type III bursts, Geophys. Res. Lett., 5, 881-884, 1978.

Papadopoulos, K., Goldstein, M. L., and Smith, R. A.: Stabilization of electron streams in type III solar radio bursts, Astrophys. J., 190, 175-186, 1974.

Robinson, P. A. and Cairns, I. H.: Stochastic growth theory of type III radio bursts, Astrophys. J., 418, 506, 1993.
Sagdeev, R. Z.: The 1976 Oppenheimer lectures: Critical problems in plasma astrophysics. II. Singular layers and reconnection, Rev. Mod. Phys., 51, 11-20, 1979.

Shapiro, V. D. and Shevchenko, V. I.: Strong Turbulence of Plasma Oscillations, in: Basic Plasma Physics II, edited by: Galeev, A. A. and Sudan, R. N., p. 123, Elsewer, Amsterdam, 1985.

Smith, R. A., Goldstein, M. L., and Papadopoulos, K.: Nonlinear stability of solar type III radio bursts, I, Theory, Astrophys. J., 234, 348-362, 1979.

Sturrock, P. A.: Type III solar radio bursts, in: Proceedings of AASNASA Symposium on the Physics of Solar Flares, edited by: Hess, W. N. (NASA SP-50; Washington, D.C., Sci. and Tech. Info. Info. Div.), p. 357, 1964.

Thejappa, G. and MacDowall, R. J.: Evidence for strong and weak turbulence processes in the source region of a local type III radio burst, Astrophys. J., 498, 465-478, 1998.

Thejappa, G. and MacDowall, R. J.: High frequency ion sound waves associated with Langmuir waves in type III radio burst source regions, Nonlin. Processes Geophys., 11, 411-420, doi:10.5194/npg-11-411-2004, 2004.

Thejappa, G., Lengyel-Frey, D., Stone, R. G., and Goldstein, M. L.: Evaluation of emission mechanisms at $\omega_{\text {pe }}$ using Ulysses observations of type III bursts, Astrophys. J., 416, 831-844, 1993.

Thejappa, G., Stone, R. G., and Goldstein, M. L.: Detection of Langmuir solitons: implications for type III burst emission mechanisms at $2 \omega_{\text {pe }}$, Astrophys. Space Sci., 243, 195-198, 1996.

Thejappa, G., Goldstein, M. L., MacDowall, R. J., Papadopoulos, K., and Stone, R. G.: Evidence for Langmuir envelope solitons in solar type III radio burst source regions, J. Geophys. Res., 104, 28279-28293, 1999.

Thejappa, G., MacDowall, R. J.,Scime, E. E., and Littleton, J. E.: Evidence for electrostatic decay in the solar wind at 5.2 AU, J. Geophys. Res., 108, 1139, doi:10.1029/2002JA009290, 2003.

Thejappa, G., MacDowall, R. J., Bergamo, M., and Papadopoulos, K.: Evidence for the oscillating two stream instability and spatial collapse of Langmuir waves in a solar type III radio burst, Astrophys. J., 747, L1-L4, doi:10.1088/2041-8205/747/1/L1, 2012a.

Thejappa, G., MacDowall, R. J., and Bergamo, M.: Phase coupling in Langmuir wave packets: Evidence of four wave interactions in solar type III radio bursts, Geophys. Res. Lett., 39, L05103, doi:10.1029/2012GL051017, 2012b.

Thejappa, G., MacDowall, R. J., and Bergamo, M.: In situ detection of strong Langmuir turbulence processes in solar type III radio bursts J. Geophys. Res., 117, A08111, doi:10.1029/2012JA017695, 2012c.

Thejappa, G., MacDowall, R. J., and Bergamo, M.: Detection of Collapsing Langmuir Wave Packets in Solar Type III Radio Bursts, AIP Conf. Proc., 1539, 70, doi:10.1063/1.4810992, 2013a.

Thejappa, G., MacDowall, R. J., and Bergamo, M.: Observational Evidence for the Collapsing Langmuir Wave Packet in a Solar Type III Radio Burst, J. Geophys. Res., 118, doi:10.1002/jgra.50441, 2013b.

Thornhill, S. G. and ter Haar, D.: Langmuir turbulence and modulational instability, Phys. Rep., 43, 43-99, 1978.

Walker, S. N., Balikhin, M. A., Bates, I., and Huff, R.: An investigation into instrumental nonlinear effects, Adv. Space Res., 30, 2815-2820, 2002. 
Zakharov, V. E.: Collapse of Langmuir waves, Sov. Phys.-JETP, 35, 908-914, 1972.

Zakharov, V. E.: Collapse and self-focusing of Langmuir waves, in: Basic Plasma Physics II, edited by: Galeev, A. A. and Sudan, R. N., p. 81, Elsevier, Amsterdam, 1985.
Zheleznyakov, V. V.: Electromagnetic Waves in a Cosmic Plasma, Nauka, Moscow, 1976 (in Russian). 